\title{
Synthesis and some properties of three stereoisomers of 1,4-diethoxy-2,5-bis[2-(thien-2-yl)ethenyl]benzene
}

\author{
Rafał Gabański, Krzysztof Waśkiewicz, and Jerzy Suwiński* \\ Faculty of Chemistry, Silesian University of Technology, 44-100 Gliwice, Poland \\ E-mail: Jerzy.Suwinski@polsl.pl
}

This paper is dedicated to Professor Lŭbor Fišera - a good colleague and a friend on the occasion of his $60^{\text {th }}$ birthday

(received 21 Oct 04; accepted 19 Dec 04; published on the web 22 Dec 04)

\begin{abstract}
The title electroluminescent macromonomers were synthesized starting from hydroquinone in four following steps: $O$-ethylation, $C$-chloromethylation, reaction with triphenylphosphine and Wittig's condensation of the resulting salt with 2-thienylcarbaldehyde. The product was separated into three stereoisomers. Structures of the stereoisomers were studied by spectroscopy and rentgenography as well as by electrochemical methods.
\end{abstract}

Keywords: Thienyl-PPV derivatives, synthesis, stereoisomers, x-ray structure

\section{Introduction}

Interesting optical properties, among them electroluminescence, of conjugated macromonomers and polymers of linear structure have recently focused a wide attention to those molecules. ${ }^{1,2}$ For that type of substances a correlation between the molecular structure and both optical and electrical properties is fundamental, when any applications of these systems are considered in e.g., optoelectronics or molecular electronics. ${ }^{2,3}$ Macromonomers that have exactly defined structure are excellent model systems that can be used to study basic properties of the corresponding polymers. In this paper we demonstrate a synthesis of three stereoisomers of 1,4diethoxy-2,5-bis[2-(thien-2-yl)ethenyl]benzene and an effect of the stereo-structure of the newly synthesized compounds on theirs some properties. Data on electrochemical and optical behavior of the macromonomers synthesized by us and products of theirs electro-polymerization have been disclosed elsewhere. ${ }^{4}$ It is worth mentioning that in other papers concerning similar compounds the most stable $(E E)$ isomers or mixtures of stereoisomers were only reported and studied. 


\section{Results and Discussion}

The title macromonomers were synthesized in four steps starting from hydroquinone according to slightly modified known general respective procedures used in each step. (Scheme)

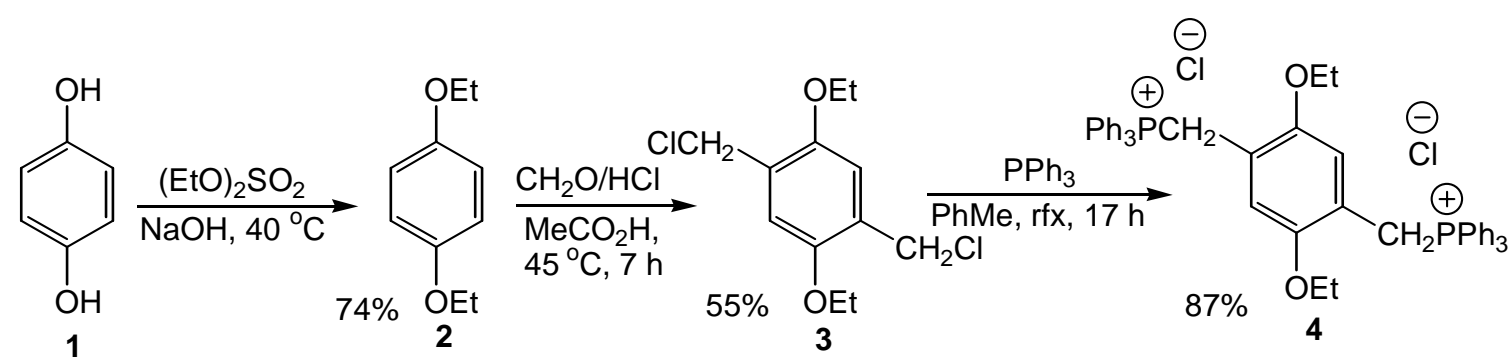

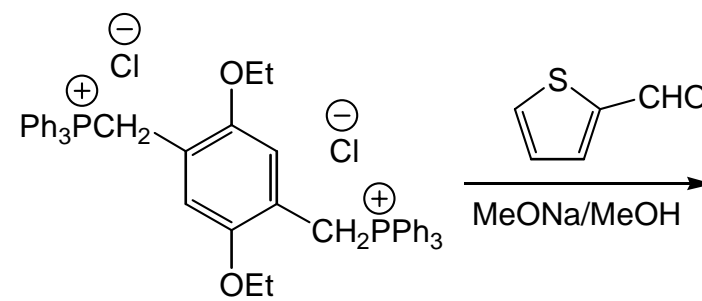

4

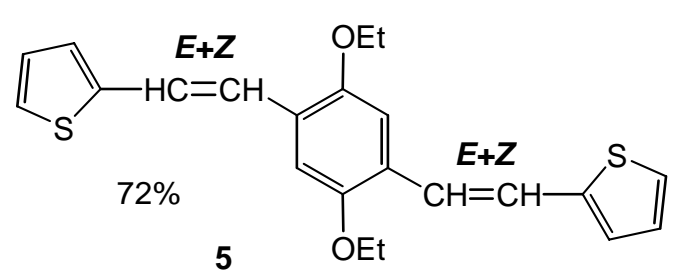

$E E: E Z: Z Z=2: 5: 3$

Scheme 1. Four-step synthesis of 1,4-diethoxy-2,5-bis[2-(thien-2-yl)ethenyl]benzene (overall yield 26\%).

Hydroquinone (1) was $O$-alkylated using diethyl sulfate. The obtained diether 2 was chloromethylated and the reaction product $\mathbf{3}$ was then treated with triphenylphosphine to produce the corresponding salt 4 , which was directly used for the Wittig's reaction ${ }^{5}$ with 2thienylcarbaldehyde to afford 1,4-diethoxy-2,5-bis[2-(thien-2-yl)ethenyl]benzene (5) as a mixture of $\mathbf{5 E E}, \mathbf{5 E Z}$ and $\mathbf{5 Z Z}$ stereoisomers (proportions 2:5:3 from ${ }^{1} \mathrm{H}$ NMR spectra). Optimized overall yield of the four-step synthesis was $26 \%$. The mixture contained three types of crystals, which were preliminarily separated by hands followed by fractional recrystallization from hexane. Overall yield of the separation was $78 \%$. Pure stereoisomers were characterized by m.p., EA, ${ }^{1} \mathrm{H}$ and ${ }^{13} \mathrm{C}$ NMR, UV-VIS as well as MS spectra. All the analytical data were consistent with the proposed structures. Additionally, the structures of $5 E E$ and $5 Z Z$ isomers were confirmed by x-ray measurements (Fig. 1 and 2). Polycrystalline $\mathbf{5 E Z}$ isomer was not suitable for crystallography. ${ }^{13} \mathrm{C}$ NMR spectra of the three isomers were also recorded in the solid state. The results of detailed analysis of those spectra and the spectra obtained in solutions will be compared with the data from x-ray crystallography in a separate paper. In the monocrystals of $\mathbf{5 E E}$ and $\mathbf{5 Z Z}$ isomers the thiophene substituents were disordered over two orientations. Attempts were made in order to distinguish between the two possibilities: the partially disordered mode as shown in Figure 1 or 2 and the structure of lower symmetry, however it did not lead to better results. 


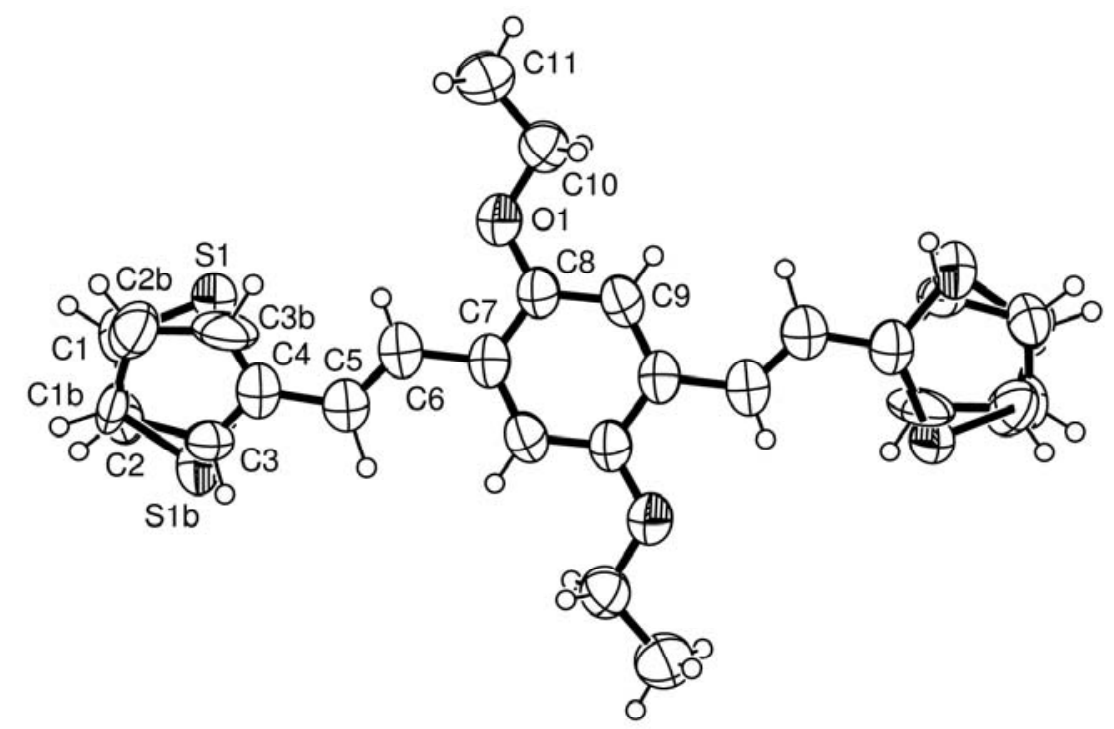

Figure 1. The molecular structure of $\mathbf{5 E E}$ isomer of 1,4-diethoxy-2,5-bis-[2-(thien-2yl)ethenyl]benzene showing 50\% probability displacement ellipsoids.

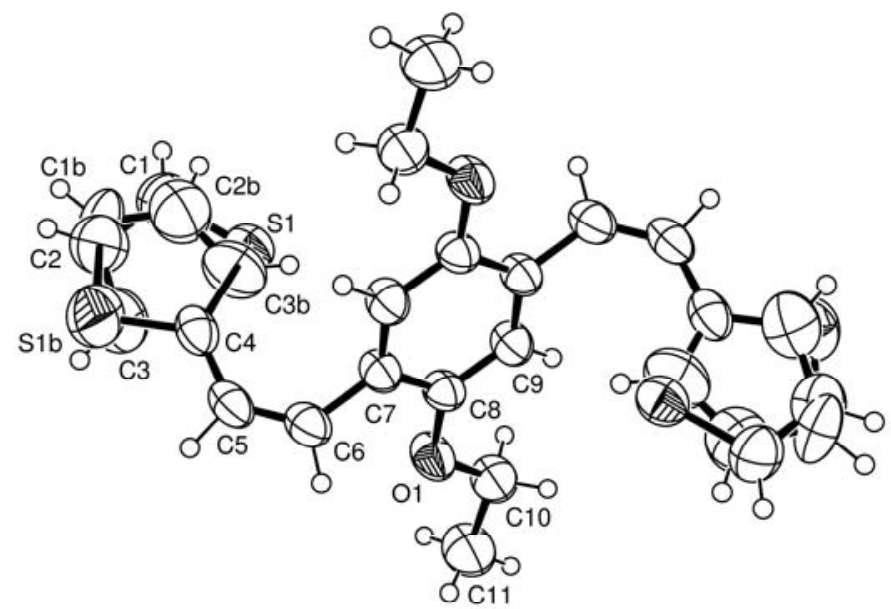

Figure 2. The molecular structure of 5ZZ isomer of 1,4-diethoxy-2,5-bis-[2-(thien-2yl)ethenyl]benzene showing 50\% probability displacement ellipsoids.

Other authors have observed similar phenomena for some thiophene derivatives too. Basic structural checkCIF/Platon ${ }^{6}$ was obtained for the results of crystal structures determination of $\mathbf{5 E E}$ and $\mathbf{5 Z Z}$ isomers. No syntax error was found. Crystallographic data have been deposited with the Cambridge Crystallographic Data Centre as supplementary publications no. 257687 (for $\mathbf{5 E E}$ ) and 257688 (for $\mathbf{5 Z Z}$ ). Some more important data concerning crystal parameters and structure refinement are also disclosed in an experimental section (Table). UV-VIS absorption spectra clearly indicated different optical properties of the stereoisomers studied $\left[\lambda_{\max } \mathbf{5 E E}=402\right.$ (in $\mathrm{CH}_{2} \mathrm{Cl}_{2}$ ), 402.6 (in $\mathrm{CH}_{3} \mathrm{CN}$ ) nm, $\lambda_{\max } \mathbf{5 E Z}=377$ (in $\mathrm{CH}_{2} \mathrm{Cl}_{2}$ ), 374.0 (in $\mathrm{CH}_{3} \mathrm{CN}$ ) nm and 
$\lambda_{\max } \mathbf{Z Z}=345$ (in $\mathrm{CH}_{2} \mathrm{Cl}_{2}$ ), 347.4 (in $\mathrm{CH}_{3} \mathrm{CN}$ ) nm]. The highest absorbance at the longest wavelength was recorded for $\mathbf{5 E E}$ molecule (see the experimental part). The photoluminescence spectra were obtained in dichloromethane solutions at concentrations of $0.05 \mathrm{mM}$ under excitation at the wavelength of the main absorption band and are published elsewhere ${ }^{4}$. In each spectrum two main bands at $460 \mathrm{~nm}$ and $490 \mathrm{~nm}$ were present with intensities decreasing in the following order $5 E E, 5 E Z$ and $5 Z Z$.

Table 1. Crystal data and structure refinement for $\mathbf{5 E E}$ and $5 Z Z$

\begin{tabular}{|c|c|c|}
\hline \multicolumn{3}{|l|}{ Crystal data } \\
\hline & $5 E E$ & $5 Z Z$ \\
\hline Chemical formula & $\mathrm{C}_{22} \mathrm{H}_{22} \mathrm{O}_{2} \mathrm{~S}_{2}$ & $\mathrm{C}_{22} \mathrm{H}_{22} \mathrm{O}_{2} \mathrm{~S}_{2}$ \\
\hline $\mathrm{Mr}$ & 382.52 & 382.52 \\
\hline Cell setting, space group & Monoclinic, $\mathrm{P} 2{ }_{1} / \mathrm{n}$ & Tetragonal, $\mathrm{I} 4_{1} / \mathrm{a}$ \\
\hline $\mathrm{a}, \mathrm{b}, \mathrm{c}(\AA)$ & $\begin{array}{l}7.5896 \quad(15), \quad 15.284 \quad(3), \\
8.6894(17)\end{array}$ & $\begin{array}{l}18.652(3), 18.652(3), \\
11.901(2)\end{array}$ \\
\hline$\beta\left(\left(^{\circ}\right)\right.$ & $97.77(3)$ & 90 \\
\hline $\mathrm{V}\left(\AA^{3}\right)$ & $998.7(3)$ & $4140.1(12)$ \\
\hline $\mathrm{Z}$ & 2 & 8 \\
\hline $\mathrm{D}_{\mathrm{x}}\left(\mathrm{Mg} \mathrm{m}^{-3}\right)$ & 1.272 & 1.227 \\
\hline Radiation type & Mo K $\alpha$ & Mo K $\alpha$ \\
\hline No. of refl. for cell param. & 91 & 98 \\
\hline$\theta$ range $\left(^{\circ}\right)$ & $3-23$ & $3-23$ \\
\hline$\mu\left(\mathrm{mm}^{-1}\right)$ & 0,280 & 0.270 \\
\hline Temperature (K) & 293 & 293 \\
\hline Crystal form, colour & Plate, brown & Block, yellow \\
\hline Crystal size (mm) & $0.5 \times 0.4 \times 0.2$ & $0.4 \times 0.4 \times 0.4$ \\
\hline \multicolumn{3}{|l|}{ Data collection } \\
\hline Diffractometer & KM4 four-circle & KM4 four-circle \\
\hline Data collection method & $\omega / 2 \theta$ scans & $\omega / 2 \theta$ scans \\
\hline Absorption correction & none & none \\
\hline $\begin{array}{l}\text { No. of measured, independent } \\
\text { and observed reflections }\end{array}$ & $1888,1769,1073$ & $1963,1837,1077$ \\
\hline $\begin{array}{l}\text { Criterion for observed } \\
\text { reflections }\end{array}$ & $\mathrm{I}>2 \sigma(\mathrm{I})$ & $\mathrm{I}>2 \sigma(\mathrm{I})$ \\
\hline Rint & 0.0781 & 0.0773 \\
\hline$\theta \max$ & 25.05 & 25.09 \\
\hline \multirow[t]{3}{*}{ Range of h, k, 1} & $h=-9 \rightarrow 8$ & $\mathrm{~h}=0 \rightarrow 22$ \\
\hline & $\mathrm{k}=0 \rightarrow 18$ & $\mathrm{k}=0 \rightarrow 22$ \\
\hline & $1=0 \rightarrow 10$ & $1=0 \rightarrow 14$ \\
\hline
\end{tabular}


Table 1. Continued

\begin{tabular}{|c|c|c|}
\hline $\begin{array}{l}\text { No. and frequency of standard } \\
\text { reflections }\end{array}$ & 3 every 80 & 3 every 80 \\
\hline Intensity decay $(\%)$ & -0.99 & -0.57 \\
\hline \multicolumn{3}{|l|}{ Refinement } \\
\hline Refinement on & $\mathrm{F}^{2}$ & $\mathrm{~F}^{2}$ \\
\hline $\mathrm{R}\left[\mathrm{F}^{2}>2 \sigma\left(\mathrm{F}^{2}\right)\right], \mathrm{wR}\left(\mathrm{F}^{2}\right), \mathrm{S}$ & $0.0551,0.1632,1.026$ & $0.0335,0.0940,0.998$ \\
\hline No. of reflections & 1769 & 1837 \\
\hline No. of parameters & 171 & 187 \\
\hline $\mathrm{H}$-atom treatment & $\begin{array}{l}\text { by mixture independent and } \\
\text { constrained refinement }\end{array}$ & $\begin{array}{l}\text { by mixture independent and } \\
\text { constrained refinement }\end{array}$ \\
\hline Weighting scheme & $\mathrm{w}=1 /\left[\sigma^{2}\left(\mathrm{~F}_{\mathrm{o}}^{2}\right)+(0.1070 \mathrm{P})^{2}+\right.$ & $\mathrm{w}=1 /\left[\sigma^{2}\left(\mathrm{~F}_{\mathrm{o}}^{2}\right)+(0.0482 \mathrm{P})^{2}+\right.$ \\
\hline & $0.0000 \mathrm{P}]$ where & $0.1357 \mathrm{P}]$ where \\
\hline & $\mathrm{P}=\left(\mathrm{F}_{\mathrm{o}}^{2}+2 \mathrm{~F}_{\mathrm{c}}^{2}\right) / 3$ & $\mathrm{P}=\left(\mathrm{F}_{\mathrm{o}}^{2}+2 \mathrm{~F}_{\mathrm{c}}^{2}\right) / 3$ \\
\hline$(\Delta / \sigma) \max$ & 0.003 & 0.047 \\
\hline$\Delta \rho \max , \Delta \rho \min \left(\mathrm{e} \AA^{-3}\right)$ & $0.404,-0.269$ & $0.186,-0.124$ \\
\hline Extinction method & SHELXL & SHELXL \\
\hline Extinction coefficient & $0.042(8)$ & $0.0021(4)$ \\
\hline
\end{tabular}

\section{Conclusions}

Concluding, a model macromonomer namely 1,4-diethoxy-2,5-bis[2-(thien-2-yl)ethenyl]benzene (5) was obtained in three pure stereoisomeric $5 \boldsymbol{E E}, 5 E Z$ and $5 Z Z$ forms. The structures of $5 \boldsymbol{E} \boldsymbol{E}$ and $\mathbf{5 Z Z}$ isomers were confirmed using $\mathrm{X}$-ray measurements. Three isomers have photoluminescence properties. They undergo electrochemical polymerization. ${ }^{4}$

\section{Experimental Section}

General Procedures. Melting points (not corrected) were determined on Boetius HMK apparatus. NMR spectra were taken in $\mathrm{CDCl}_{3}$ with TMS as an internal reference by Varian XL300 at $300 \mathrm{MHz}$ for ${ }^{1} \mathrm{H}$ and at $75.5 \mathrm{MHz}$ for ${ }^{13} \mathrm{C}$. UV-VIS spectra were recorded using Shimadzu UV-2102 PC recording spectrophotometer in acetonitrile solution using kuvetes $1 \mathrm{~cm}$ long. MS spectra were obtained from HPLC-MS Integrity Systems with Termabeam Mass Detector (EI, 70 $\mathrm{eV}$ ); samples (in methanol solutions) were introduced to the spectrometer using direct inlet. Xray diffraction data were collected on KUMA KM4 four-circle diffractometer at $295 \mathrm{~K}$. 


\section{Synthesis of 1,4-diethoxy-2,5-bis[2-(thien-2-yl)ethenyl]benzenes}

Step 1. Hydroquinone (1) (35mmol) was $O$-alkylated in aqueous sodium hydroxide $(4.5 \mathrm{~g} \mathrm{NaOH}$ and $32 \mathrm{~cm}^{3}$ water)using diethyl sulfate $(140 \mathrm{mmol}){ }^{7}$ The mixture was heated under nitrogen at $40{ }^{\circ} \mathrm{C}$ for $1.5 \mathrm{~h}$ and a product was steam distilled to give a crude diether 2: $74 \%$, m.p. $71-72{ }^{\circ} \mathrm{C}$ (lit. $\left.71-72{ }^{\circ} \mathrm{C}\right)^{8},{ }^{1} \mathrm{H} \mathrm{NMR}\left(\mathrm{CDCl}_{3}, \delta \mathrm{ppm}\right): 1.39(6 \mathrm{H}, \mathrm{t}, \mathrm{J}=7.05 \mathrm{~Hz}), 3.96(4 \mathrm{H}, \mathrm{q}, \mathrm{J}=7.00 \mathrm{~Hz})$, $6.81(4 \mathrm{H}, \mathrm{s})$.

Step 2. A mixture of crude $2(6 \mathrm{mmol})$, glacial acetic acid $\left(5.45 \mathrm{~cm}^{3}\right)$, concentrated hydrochloric acid $\left(6.36 \mathrm{~cm}^{3}\right)$ and paraformaldehyde $(16 \mathrm{mmol})$ was saturated with gaseous hydrochloride at $45{ }^{\circ} \mathrm{C}$ for $7 \mathrm{~h}$. The solids were collected, rinsed with water and crystallized from chloroform to give product 3: 55\%, m.p. $153-154.5{ }^{\circ} \mathrm{C}$, (lit. $\left.148-151{ }^{\circ} \mathrm{C}\right)^{9},{ }^{1} \mathrm{H} \mathrm{NMR}\left(\mathrm{CDCl}_{3}, \delta \mathrm{ppm}\right): 1.42(6 \mathrm{H}$, $\mathrm{t}, \mathrm{J}=6.9 \mathrm{~Hz}), 4.06(4 \mathrm{H}, \mathrm{q}, \mathrm{J}=7 \mathrm{~Hz}), 4.63(4 \mathrm{H}, \mathrm{s}), 6.91(2 \mathrm{H}, \mathrm{s}) ;{ }^{13} \mathrm{C} \mathrm{NMR}\left(\mathrm{CDCl}_{3}, \delta \mathrm{ppm}\right): 14.92$ $\left(\mathrm{CH}_{3}\right), 41.29\left(\mathrm{CH}_{2} \mathrm{Cl}\right), 64.77\left(\mathrm{OCH}_{2}\right), 114.50(\mathrm{Ar}, \mathrm{CH}), 127.15\left(\mathrm{Ar}, \mathrm{CCH}_{2}\right), 150.46$ (Ar, COEt).

Step 3. Compound $3(9.5 \mathrm{mmol})$ was treated with triphenylphosphine $(21 \mathrm{mmol})$ in dry refluxing toluene for $17 \mathrm{~h}$ to afford on cooling precipitations of the corresponding phosphonium salt. The precipitations were collected, washed with anhydrous diethyl ether and dried under diminished pressure to give product 4: 87\%, m.p. $264-265{ }^{\circ} \mathrm{C},{ }^{1} \mathrm{HNMR},\left(\mathrm{CDCl}_{3}, \delta \mathrm{ppm}\right): 0.77\left(6 \mathrm{H}, \mathrm{CH}_{3}, \mathrm{t}, \mathrm{J}\right.$ $=6.9 \mathrm{~Hz}), 3.12\left(4 \mathrm{H}, \mathrm{CH}_{2}, \mathrm{q}, \mathrm{J}=6.9 \mathrm{~Hz}\right), 5.31\left(4 \mathrm{H}, \mathrm{Ar}-\mathrm{CH}_{2}, \mathrm{~d},{ }^{2} \mathrm{~J}_{\mathrm{H}-\mathrm{P}}=12.9 \mathrm{~Hz}\right), 6.79(\mathrm{Ar}, 2 \mathrm{H}, \mathrm{s})$, 7.58-7.80 (Ar, 3H, 2m). ${ }^{13} \mathrm{CNMR},\left(\mathrm{CDCl}_{3}, \delta \mathrm{ppm}\right): 14.31\left(\mathrm{CH}_{3}\right), 25.15\left(\mathrm{PhCH}_{2} \mathrm{PPh}_{3}, \mathrm{~d},{ }^{1} \mathrm{~J}_{\mathrm{P}-\mathrm{C}}=\right.$ $47.2 \mathrm{~Hz}), 63.58\left(\mathrm{OCH}_{2}\right), 116.19(\mathrm{Ar}, \mathrm{CH}), 116.4\left(\mathrm{Ar}, \mathrm{C}-\mathrm{CH}_{2}-\right), 118.19\left(\mathrm{PPh}_{3}, \mathrm{C} 1,{ }^{1} \mathrm{~J}_{\mathrm{P}-\mathrm{C}}=\right.$ 86.1Hz), 130.13, 130.05, $129.97\left(\mathrm{PPh}_{3}, \mathrm{C} 1, \mathrm{~m}\right), 134.37,134.31,134.25\left(\mathrm{PPh}_{3}, \mathrm{C} 2, \mathrm{~m}\right) ; 134.81$ $\left(\mathrm{PPh}_{3} \mathrm{C} 4\right), 150.42$ (Ar, C-O-). It was pure enough for the next step.

Step 4. Salt 4 and 2-thienylcarbaldehyde ${ }^{5}$ were used for the Wittig reaction performed in the inert anhydrous atmosphere. Sodium methoxide solution in methanol (obtained by dissolving 9.0 mmol of sodium in $15 \mathrm{ml}$ of methanol) was added gradually within $1 \mathrm{~h}$ without heating to a stirred mixture of 2-thienylcarbaldehyde $(9.1 \mathrm{mmol})$, phosphonium salt $4(4.4 \mathrm{mmol})$ and anhydrous methanol $(45 \mathrm{ml})$. The reaction mixture was refluxed for $7 \mathrm{~h}$ and then left without heating for $18 \mathrm{~h}$. The solvents were evaporated to dryness and the residues were extracted with benzene-hexane $(1: 1, \mathrm{v} / \mathrm{v})$. Triphenylphospine oxide was removed from the obtained solution by chromatography. Evaporation of the remaining solution gave a mixture $(72 \%)$ of almost pure $\mathbf{5 E E}, \mathbf{5 E Z}$ and $\mathbf{5 Z Z}$ stereoisomers of 1,4-diethoxy-2,5-bis[2-(thien-2-yl)ethenyl]benzenes (2:5:3 from ${ }^{1} \mathrm{H}$ NMR measurements). Overall yield of the four-step synthesis of 5 was c.a. $26 \%$. The mixture contained three types of crystals, which were preliminarily separated by hands followed by two fractional crystallizations from hexane. Overall yield of the separation was $78 \%$. Pure stereoisomers were characterized by m.p.s, elementary analyses, ${ }^{1} \mathrm{H},{ }^{13} \mathrm{C} \mathrm{NMR}$, UV and MS spectra. Structures of $5 \mathbf{E E}$ and $\mathbf{5 Z Z}$ isomers were additionally confirmed by x-ray measurements.

Data common for three isomers of 1,4-diethoxy-2,5-bis[2-(thien-2-yl)ethenyl]benzene (5). EA calc. for $\mathrm{C}_{22} \mathrm{H}_{22} \mathrm{O}_{2} \mathrm{~S}_{2} \% \mathrm{C} 69.07, \% \mathrm{H}$ 5.82, \%S 16,76; Found: \%C 68.86-68.99, \%H 5.825.87, \%S 16.65-16.70. MS (70eV, m/z, \%): M calc. 382.54; Found: 384,1 (M+2, 9-15), 383,2 (M+1, 26-27), 382,2 (M+100), 353.3 (M-Et, 7-9\%), 324.1 (M-2Et, 8-11), 229.1 (8-10), 213.1 (5-6), $191.2\left(\mathrm{M}^{++}, 3-7\right)$ and others of lower $\mathrm{m} / \mathrm{z}$. 
Additional data. $5 \boldsymbol{E E}$ isomer: m. p. $174.5-176{ }^{\circ} \mathrm{C} ;{ }^{1} \mathrm{HNMR}\left(\mathrm{CDCl}_{3}, \delta \mathrm{ppm}\right): 1.48\left(2 \mathrm{CH}_{3}, \mathrm{t}, \mathrm{J}=\right.$ $7 \mathrm{~Hz}), 4.10\left(2 \mathrm{CH}_{2}, \mathrm{q}, \mathrm{J}=7 \mathrm{~Hz}\right), 7.02(\mathrm{Ar}, 2 \mathrm{C}-\mathrm{H}, \mathrm{s}), 7.24(2=\mathrm{CH}, \mathrm{d}, \mathrm{J}=16.2 \mathrm{~Hz}), 7.28(2$ $=\mathrm{CH}, \mathrm{d}, \mathrm{J}=16.2 \mathrm{~Hz}), 7.00\left(\mathrm{C}_{4} \mathrm{H}_{3} \mathrm{~S}, 2 \mathrm{C}_{4}-\mathrm{H}, \mathrm{dd}, \mathrm{J}=3.6 \mathrm{~Hz}, \mathrm{~J}=5.1 \mathrm{~Hz}\right), 7.06\left(\mathrm{C}_{4} \mathrm{H}_{3} \mathrm{~S}, 2 \mathrm{C}_{3}-\mathrm{H}, \mathrm{d}\right.$, $\left.\mathrm{J}=3.6 \mathrm{~Hz}), 7.18\left(\mathrm{C}_{4} \mathrm{H}_{3} \mathrm{~S}, 2 \mathrm{C}_{5} \mathrm{H}, \mathrm{d}, \mathrm{J}=5.1 \mathrm{~Hz}\right),{ }^{13} \mathrm{CNMR}\left(\mathrm{CDCl}_{3}\right), \delta \mathrm{ppm}\right): 150.90,143.75$, $127.58,126.43,125.73,124.20,123.38,122.16,110.79,65.04,15.03$; UV in MeCN: $(\lambda \mathrm{nm}, \varepsilon$ $\left.\left[\mathrm{dm}^{3} / \mathrm{mol} \mathrm{cm}\right]\right): 402.6,46350$.

5EZ isomer. $99-100{ }^{\circ} \mathrm{C} ;{ }^{1} \mathrm{HNMR},\left(\mathrm{CDCl}_{3}, \delta \mathrm{ppm}\right): 1.33-1.41\left(2 \mathrm{CH}_{3}, \mathrm{~m}\right), 3.91\left(\mathrm{CH}_{2}, \mathrm{q}, \mathrm{J}=7\right.$ $\mathrm{Hz}), 4.04\left(\mathrm{CH}_{2}, \mathrm{q}, \mathrm{J}=7 \mathrm{~Hz}\right), 6.56(=\mathrm{CH}, \mathrm{d}, \mathrm{J}=12 \mathrm{~Hz}), 6.71(=\mathrm{CH}, \mathrm{d}, \mathrm{J}=12 \mathrm{~Hz}), 7.26$ (=CH, d, J $=16.2 \mathrm{~Hz}), 7.30(=\mathrm{CH}, \mathrm{d}, \mathrm{J}=16.2 \mathrm{~Hz}), 6.89\left(\mathrm{C}_{4} \mathrm{H}_{3} \mathrm{~S}, \mathrm{C}_{4}-\mathrm{H}, \mathrm{dd}, \mathrm{J}=3.6 \mathrm{~Hz}, \mathrm{~J}=5.1 \mathrm{~Hz}\right), 6.97-$ $7.03\left(\mathrm{C}_{4} \mathrm{H}_{3} \mathrm{~S}, \mathrm{C}_{3}-\mathrm{H}, \mathrm{Ar}, \mathrm{C}-\mathrm{H}, \mathrm{C}_{4} \mathrm{H}_{3} \mathrm{~S}, \mathrm{C}_{5}-\mathrm{H}, \mathrm{m}\right)$, 7.04-7.07 $\left(\mathrm{C}_{4} \mathrm{H}_{3} \mathrm{~S}, \mathrm{C}_{3}-\mathrm{H}, \mathrm{Ar}, \mathrm{C}-\mathrm{H}, \mathrm{m}\right), 7.09$ $\left(\mathrm{C}_{4} \mathrm{H}_{3} \mathrm{~S}, \mathrm{C}_{5}-\mathrm{H}, \mathrm{d}, \mathrm{J}=5.1 \mathrm{~Hz}\right), 7.17\left(\mathrm{C}_{4} \mathrm{H}_{3} \mathrm{~S}, \mathrm{C}_{5}-\mathrm{H}, \mathrm{d}, \mathrm{J}=5.1 \mathrm{~Hz}\right) ;{ }^{13} \mathrm{CNMR},\left(\mathrm{CDCl}_{3}, \delta \mathrm{ppm}\right)$ : $150.91,150.31,143.75,140.12,128.20,127.55,126.52,126.38,125.69,125.33,124.72,124.16$, $123.57,123.22,122.22,115.06,110.68,64.88,64.80,14.99 ; \mathrm{UV}$ in $\mathrm{MeCN}:(\lambda \mathrm{nm}, \varepsilon$ $\left.\left[\mathrm{dm}^{3} / \mathrm{mol} \mathrm{cm}\right]\right): 374.0,26200$.

5ZZ isomer. $129-130{ }^{\circ} \mathrm{C}$; ${ }^{1} \mathrm{H}$ NMR $\left(\mathrm{CDCl}_{3}, \delta\right.$ ppm): $1.26\left(2 \mathrm{CH}_{3}, \mathrm{t}, \mathrm{J}=7 \mathrm{~Hz}\right), 3.87\left(2 \mathrm{CH}_{2}, \mathrm{q}, \mathrm{J}\right.$ $=7 \mathrm{~Hz}), 6.58(2=\mathrm{CH}, \mathrm{d}, \mathrm{J}=12 \mathrm{~Hz}), 6.73(2=\mathrm{CH}, \mathrm{d}, \mathrm{J}=12 \mathrm{~Hz}), 6.90\left(\mathrm{C}_{4} \mathrm{H}_{3} \mathrm{~S}, 2 \mathrm{C}-\mathrm{H}, \mathrm{dd}, \mathrm{J}=\right.$ $3.6 \mathrm{~Hz}, \mathrm{~J}=5.1 \mathrm{~Hz}), 7.01\left(\mathrm{Ar}, 2 \mathrm{C}-\mathrm{H}, \mathrm{C}_{4} \mathrm{H}_{3} \mathrm{~S}, \mathrm{C}_{3}-\mathrm{H}, \mathrm{s}\right), 7.03\left(\mathrm{C}_{4} \mathrm{H}_{3} \mathrm{~S}, \mathrm{C}_{3}-\mathrm{H}, \mathrm{s}\right), 7.10\left(\mathrm{C}_{4} \mathrm{H}_{3} \mathrm{~S}, 2 \mathrm{C}_{5^{-}}\right.$ $\mathrm{H}, \mathrm{d}, \mathrm{J}=5.1 \mathrm{~Hz}) ;{ }^{13} \mathrm{CNMR},\left(\mathrm{CDCl}_{3}, \delta \mathrm{ppm}\right): 150.23,140.13,128.17,126.60,126.29,125.24$, 124.83, 123.23, 114.86, 64.62, 14.91; UV in MeCN: $\left(\lambda \mathrm{nm}, \varepsilon\left[\mathrm{dm}^{3} / \mathrm{mol} \mathrm{cm}\right]\right): 347.4,11750$.

\section{X-ray measurements}

Monocrystals of $\mathbf{5 E E}$ and $\mathbf{5 Z Z}$ were obtained by a slow evaporation of hexane solutions of the preliminary separated isomers. Diffraction data were collected using MoK $\alpha$ radiation (0.71073 $\AA$ ) on a four-circle KUMA KM-4 diffractometer with a graphite monochromator at $295 \mathrm{~K}$ using $\omega-2 \theta$ scan. Cell parameters, reflections collection and theirs reduction were done using KUMA KM4 software. ${ }^{10}$ No absorption correction was introduced. The structures were determined by means of direct methods and refined by the full-matrix least squares technique. ${ }^{11}$ Position of non - hydrogen atoms were determined using anisotropic thermal parameters. Most of hydrogen atoms were found in subsequent difference Fourier maps and isotropically refined; thiophene hydrogen atoms were added. Data concerning crystal and structure refinement is disclosed in Table. Crystallographic data can be obtained free of charge from The Cambridge Crystallographic Data Centre via www.ccdc.cam.ac.uk/data_request/cif. For examples of thiophene ring disorders over two orientations see e.g., ref. ${ }^{12}$

\section{References}

1. (a) Burroughes, J. H.; Bradley, D. D. C.; Brown, A. R.; Marks, R. N.; Mackay, K.; Friend, R. H.; Burn, P. L.; Holmes, A. B. Nature 1990, 347, 539. (b) Van Der Looy, J. F. A.; Thys, G. J. 
H.; Dieltiens, P. E. M.; De Schrijver, D.; Van Alsenoy, C.; Geise, H. J. Tetrahedron 1997, $44,15069$.

2. (a) Gustafsson, G.; Cao,Y.; Treacy, G. M.; Klavetter, F.; Colaneri, N.; Heeger, A. J. Nature 1992, 357, 477. (b) Schwartz, B. J. Annu. Rev. Phys. Chem. 2003, 54, 141. (c) Hide, F.; DiazGarcia, M. A.; Schwartz, B. J.; Heeger, A. J. Acc. Chem. Res. 1997, 30, 430. (d) Hong-Ku Shim, Jung-Il Jin Adv. Polym. Sci. 2002, 158, 193. (e) Roncali, Annu J. Rep. Prog. Chem., Sect. C 1999, 95, 47.

3. (a) Friend, R. H. et al. Nature (London) 1999, 397, 121. (b) Baldo, M. A.; Thompson, M.; Forrest, S. R. Nature (London) 2000, 403, 750.

4. Waskiewicz, K.; Gabanski, R.; Zak, J.; Lapkowski, M.; Suwinski, J. Electrochemical and Solid State Letters 2005, 8 (2), in press.

5. Cresp, T. M.; Giles, R. G. F.; Sargent, M. V.; Brown, C.; Smith, D. O’N. J.C.S. Perkin I 1974, 21, 2435.

6. http://journals.iucr.org/c/services/authorservices.html ; Acta Crystallographica Section C: Crystal Structure Communications, checkCIF/PLATON service.

7. Preparatyka Organiczna $2^{\text {nd }}$ Edn; Bochwic, B., Ed., Warszawa: PWN, 1975; p 225.

8. Sakakibara Nippon Kagaku Zasshi 1960, 81, 495; acc. to Beilstein Information System, 1988-2004.

9. Sakamoto, K.; Ōki, M. Bull. Chem. Soc. Jap. 1975, 48, 497.

10. Kuma Diffraction, KM-4 Users Guide. Ver. KM4B8, 1997 Wroclaw, Poland.

11. Sheldrick, G. M.; SHELXS-97 and SHELXL-97, Program for the Refinement of Crystal Structures. Univ. of Göttingen: Germany 1997.

12. Irngartinger H.; Lichtenhaler J.; Herpich R., Struct. Cem. 1994, 5, 283 (acc. to Cambridge Crystallographic Data Centre). 